Article

\title{
Emergent HIV-1 Drug Resistance Mutations Were Not Present at Low-Frequency at Baseline in Non-Nucleoside Reverse Transcriptase Inhibitor-Treated Subjects in the STaR Study
}

\author{
Danielle P. Porter ${ }^{1, *}$, Martin Daeumer ${ }^{2}$, Alexander Thielen ${ }^{2}$, Silvia Chang ${ }^{1}$, Ross Martin ${ }^{1}$, \\ Cal Cohen ${ }^{1}$, Michael D. Miller ${ }^{1}$ and Kirsten L. White ${ }^{1}$ \\ Received: 29 September 2015; Accepted: 20 November 2015; Published: 7 December 2015 \\ Academic Editors: Johnson Mak, Peter Walker and Marcus Thomas Gilbert \\ 1 Gilead Sciences, 333 Lakeside Drive, Foster City, CA 94404, USA; silvia.chang@gilead.com (S.C.); \\ ross.martin@gilead.com (R.M.); cal.cohen@gilead.com (C.C.); michael.miller@gilead.com (M.D.M.); \\ kirsten.white@gilead.com (K.L.W.) \\ 2 Seq-IT GmbH \& Co. KG, Pfaffplatz 10, 67655 Kaiserslautern, Germany; \\ m.daeumer@immungenetik-kl.de (M.D.); a.thielen@immungenetik-kl.de (A.T.) \\ * Correspondence: danielle.porter@gilead.com; Tel.: +1-650-522-1368; Fax: +1-650-524-9470
}

\begin{abstract}
At Week 96 of the Single-Tablet Regimen (STaR) study, more treatment-naïve subjects that received rilpivirine/emtricitabine/tenofovir DF (RPV/FTC/TDF) developed resistance mutations compared to those treated with efavirenz (EFV)/FTC/TDF by population sequencing. Furthermore, more RPV/FTC/TDF-treated subjects with baseline HIV-1 RNA $>100,000$ copies/mL developed resistance compared to subjects with baseline HIV-1 RNA $\leqslant 100,000$ copies/mL. Here, deep sequencing was utilized to assess the presence of pre-existing low-frequency variants in subjects with and without resistance development in the STaR study. Deep sequencing (Illumina MiSeq) was performed on baseline and virologic failure samples for all subjects analyzed for resistance by population sequencing during the clinical study $(n=33)$, as well as baseline samples from control subjects with virologic response $(n=118)$. Primary NRTI or NNRTI drug resistance mutations present at low frequency ( $\geqslant 2 \%$ to $20 \%$ ) were detected in $6.6 \%$ of baseline samples by deep sequencing, all of which occurred in control subjects. Deep sequencing results were generally consistent with population sequencing but detected additional primary NNRTI and NRTI resistance mutations at virologic failure in seven samples. HIV-1 drug resistance mutations emerging while on RPV/FTC/TDF or $\mathrm{EFV} / \mathrm{FTC} / \mathrm{TDF}$ treatment were not present at low frequency at baseline in the STaR study.
\end{abstract}

Keywords: rilpivirine; efavirenz; resistance; virologic failure; minority variants

\section{Introduction}

Drug resistance testing for HIV-infected patients is recommended by treatment guidelines as part of standard of care to inform the selection of initial, as well as next-line treatment strategies after virologic failure [1]. Standard genotyping assays use population sequencing, which detects mutations present in the majority of the virus quasispecies (approximately $\geqslant 20 \%$ of the virus population). Deep sequencing (or next-generation sequencing; NGS) technologies enable the detection of low-frequency (minority) quasispecies down to a threshold of $\sim 1 \%-2 \%$ of the virus population. The clinical significance of low-frequency viral variants on treatment response is unclear. Some studies have shown an association between the presence of pre-existing low-frequency drug resistant variants with an increased risk of virologic failure, particularly for non-nucleoside reverse transcriptase inhibitors 
(NNRTIs), while other studies have shown no significant impact of low-frequency variants on virologic outcome [2-11].

The STaR study (GS-US-264-0110) was a randomized, open-label, 96-week study designed to directly compare the safety and efficacy of the two NNRTI-based single tablet regimens (STRs) rilpivirine (RPV)/emtricitabine (FTC)/tenofovir disoproxil fumarate (TDF) and efavirenz (EFV)/FTC/TDF in treatment-naïve HIV-1-infected adults [12,13]. At Week 96, resistance development to at least one component of RPV/FTC/TDF occurred more frequently than resistance development to EFV/FTC/TDF (5.3\% vs. 1.0\%, respectively). Furthermore, within the RPV/FTC/TDF arm, resistance development was more frequent in subjects with baseline HIV-1 RNA $>100,000$ copies / $\mathrm{mL}$ compared to baseline HIV-1 RNA $\leqslant 100,000$ copies $/ \mathrm{mL}$ (9.0\% vs. 3.5\%, respectively) [14]. These results are consistent with the current indication for RPV/FTC/TDF in treatment-naive patients, which is restricted to use in patients with HIV-1 RNA $\leqslant 100,000$ copies $/ \mathrm{mL}$ due to an increased risk of virologic failure and resistance development in patients with high baseline viral load $[15,16]$.

To investigate the possibility that low-frequency mutant viruses were present at baseline in subjects that experienced virologic failure with resistance development in the STaR study, deep sequencing was performed on baseline samples from these subjects compared to control subjects that were virologic responders. Virologic failure samples from subjects that developed resistance were also analyzed to further characterize the resistance patterns that emerged.

\section{Materials and Methods}

\subsection{STaR Study Design}

Details of the STaR study have been previously published [12,13]. Briefly, STaR (GS-US-264-0110; clinicaltrials.gov identifier: NCT01309243) was a phase 3b, randomized, open-label, multi-center, international, 96-week study evaluating the safety and efficacy of the RPV/FTC/TDF STR compared to the EFV/FTC/TDF STR in treatment-naïve HIV-1 infected subjects. Subjects were randomized 1:1 to RPV/FTC/TDF or EFV/FTC/TDF. Eligibility criteria included screening HIV-1 RNA $\geqslant 2500$ copies $/ \mathrm{mL}$, no prior ARV therapy, genotypic sensitivity to EFV, FTC, and TDF, and lack of the RPV mutations K101E/P, E138A/G/K/Q/R, Y181C/I/V, and H221Y by population sequencing. Randomization was stratified by screening HIV-1 RNA ( $\leqslant 100,000$ or $>100,000$ copies $/ \mathrm{mL}$ ).

\subsection{FDA Snapshot Outcome}

The primary endpoint of the STaR study was the proportion of subjects with HIV-1 RNA $<50$ copies $/ \mathrm{ml}$ at Week 48 by the FDA snapshot algorithm [12]. Efficacy by FDA snapshot algorithm at Week 96 (end of study) was a secondary objective of the study [13]. According to the FDA snapshot algorithm, results were classified as virologic success if the subject's HIV-1 RNA was $<50$ copies $/ \mathrm{mL}$ within the visit window. Results were classified as virologic failure if a subject's HIV-1 RNA was $\geqslant 50$ copies $/ \mathrm{mL}$, the subject had discontinued study drug due to lack of efficacy per the investigator's assessment, or the subject discontinued due to reasons other than adverse event or death and the last on-study drug HIV-1 RNA was $\geqslant 50$ copies $/ \mathrm{mL}$. Results were classified as no data in the visit window if a subject discontinued due to adverse event or death prior to the visit window, the subject discontinued due to reasons other than adverse event or death and the last on-study drug HIV-1 RNA was $<50$ copies $/ \mathrm{mL}$, or the subject was on study but had missing data in the visit window [17].

\subsection{Resistance Analysis Population (RAP)}

The resistance analysis population (RAP) included all subjects who qualified for resistance analysis through Week 96 of the STaR study. Subjects with suboptimal virologic response or virologic rebound (as defined below) and HIV-1 RNA $\geqslant 400$ copies $/ \mathrm{mL}$ who were on study drugs were considered to have virologic failure and were included in the RAP. Suboptimal virologic response was assessed at Week 8 and was defined as having HIV-1 RNA $\geqslant 50$ copies $/ \mathrm{mL}$ and $<1 \log _{10}$ reduction from baseline at the 
Week 8 visit, which was confirmed at the subsequent visit. Virologic rebound was defined as having two consecutive visits with HIV-1 RNA $\geqslant 50$ copies/mL after achieving HIV-1 RNA $<50$ copies/mL, or as having two consecutive visits with $>1 \log _{10}$ increase in HIV-1 RNA from nadir. The sample from the confirmation visit (if available) was analyzed for resistance development. In addition, subjects who were on study drugs, had not been analyzed previously, and had HIV-1 RNA $\geqslant 400$ copies $/ \mathrm{mL}$ at Week 48, Week 96, or their last visit (at or after Week 8) were also included in the RAP. Population sequencing results for subjects in the RAP have been previously reported in detail $[14,18]$.

\subsection{Deep Sequencing Sample Selection}

A total of 184 samples were analyzed using deep sequencing. These included 33 baseline and 33 virologic failure time point samples from subjects that met the criteria for inclusion in the RAP through Week 96 (24 RPV /FTC/TDF, 9 EFV /FTC/TDF). In addition, 118 baseline samples from control subjects with virologic response that did not qualify for the RAP (44 RPV/FTC/TDF, 74 EFV/FTC/TDF) were also analyzed. Non-RAP control subjects were chosen as a representative population matched with RAP subjects based on baseline HIV-1 RNA and CD4 cell count.

\subsection{Virology Assessments}

\subsubsection{Deep Sequencing Assay}

Genotypic resistance testing was performed using the "deepTypeHIV" assay (SeqIT GmbH \& Co. KG, Kaiserslautern, Germany). Viral RNA was isolated from patient plasma using QIAamp viral RNA mini kit (Qiagen, Hilden, Germany) according to the manufacturer's protocol. Reverse transcription and polymerase chain reaction (PCR) were carried out using SuperScript III One-Step RT-PCR System with Platinum ${ }^{\circledR}$ Taq High Fidelity (Life Technologies, Darmstadt, Germany) and primers 1RES, 5'-GAAGAAATGATGACAGCATGTCAGGG-3' (nt 1819-1844, HXB2 numbering) and 2RES, 5'-TAATTTATCTACTTGTTCATTTCCTCCAAT-3' (nt 4173-4202). Nested PCR was carried out with Platinum ${ }^{\circledR}$ Taq High Fidelity (Life technologies) and the following inner primer pair: RES3, 5'-AGACAGGCTAATTTTTTAGGGA-3' (nt 2074-2095) and RES4, 5'-ATGGYTCTT GATAAATTTGATATGTCC-3' (nt 3559-3585). All amplicons were purified using the Agencourt AMPure ${ }^{\circledR}$ XP system on a BioMek NX workstation (Beckman Coulter, Krefeld, Germany), quantified fluorometric on a FluoStar Optima (BMG Labtech, Ortenberg, Germany) using Quant-iT Picogreen dsDNA reagent (Life Technologies).

Library preparation for Illumina deep sequencing was done using a Nextera ${ }^{\circledR}$ XT DNA Sample Preparation and Index kit (Illumina, San Diego, CA, USA) according to the manufacturer's manual. Resulting libraries were normalized and pooled for subsequent sequencing on an Illumina MiSeq platform using the $2 \times 250$ cycle paired-end sequencing protocol.

The clinical cutoff for the "deepTypeHIV" assay is $2 \%$ based on the background signal present at mutational positions of interest [19]. The $2 \%$ cutoff for the "deepTypeHIV" assay was confirmed by sequencing mixtures of HIV-1 xxLAI containing the M184V and K65R mutations in RT with wild-type HIV- 1 xxLAI at $10 \%, 5 \%, 2 \%, 1 \%, 0.5 \%$, and $0.2 \%$ mutant frequencies.

\subsubsection{Deep Sequencing Analysis}

Analysis of deep sequencing data was performed using an internally-developed analysis pipeline where sequence reads in the form of FASTQ files were processed and aligned via a multi-step method.

To align the deep sequencing (next generation sequencing; NGS) results, a de novo assembly sequence from the baseline sample was used as the alignment sequence. The assembly sequence was created by the following steps: (1) contigs from paired-end reads were assembled using VICUNA [20]; and (2) contigs were then merged to generate an assembly sequence based on the alignment to the HIV reference sequence HIV-1 NL4-3 (Genbank Accession AF324493). Insertions and deletions (indels) were included in the assembly sequence for which the indel(s) did not result in a frameshift and were 
present in $>50 \%$ of the viral population. Nucleotide mixtures were included in the assembly sequence when present at $>15 \%$ of the viral population.

After a subject assembly sequence was created, the sequence reads were evaluated based on quality scores and aligned to the assembly sequence using MOSAIK v1.1.0017 [21]. More specifically, sequence reads were trimmed and filtered based on quality score and length. Trimming was performed for any reads with quality score $<15$, and any reads $<50$ nucleotides in length were excluded after trimming. All aligned reads were translated in-frame and changes from the reference sequence (HIV-1 NL4-3) were tabulated.

Mutations occurring at $\geqslant 2 \%$ were reported. Average coverage across primary nucleoside reverse transcriptase inhibitor (NRTI)- and NNRTI-associated resistance mutations was 18,802 reads per position for all samples tested.

\subsubsection{Population Sequencing Assay}

At screening, pre-existing resistance in the protease (PR) and reverse transcriptase (RT) portion of the pol gene was assessed by genotype (population sequencing) using the GeneSeq ${ }^{\mathrm{TM}}$ assay (Monogram Biosciences, San Francisco, CA, USA). These screening data were used for baseline resistance analyses. For post-baseline resistance analyses of subjects in the RAP, PR/RT genotyping (population sequencing) and phenotyping were performed using the PhenoSense GT ${ }^{\mathrm{TM}}$ assay (Monogram Biosciences) [22].

\subsubsection{Viral Load Assay}

Viral load (HIV-1 RNA copies/mL) was assessed from plasma using COBAS AMPLICOR Monitor Version 1.5 (Roche Diagnostics, Basel, Switzerland).

\subsubsection{Mutant Copy Number}

Mutant copy number at baseline was calculated by multiplying the deep sequencing variant frequency (\%) by the viral load (HIV-1 RNA copies/mL).

\section{Results}

Baseline characteristics of the subjects whose samples were analyzed by deep sequencing are described in Table 1. Baseline characteristics were generally similar between RAP and non-RAP control subjects as well as to the overall study population. The proportion of subjects with baseline HIV-1 RNA $>100,000$ copies / $\mathrm{mL}$ included in the deep sequencing analysis was greater than the proportion enrolled in the overall study population (56\% vs. $35 \%$, respectively) $[12,13]$.

Primary NRTI and NNRTI drug resistance mutations detected by deep sequencing at low frequency ( $\geqslant 2 \%$ to $20 \%$ ) in baseline samples and the list of mutations assessed are presented in Table 2 . No drug resistance mutations were detected at low frequency in baseline samples from RAP subjects while primary NRTI or NNRTI drug resistance mutations were detected at low frequency in $8.5 \%$ of samples from control subjects (6.6\% of all baseline samples tested). No subject had both primary NRTI and NNRTI mutations present at low frequency at baseline by deep sequencing. Using a more extensive list of mutations including the NNRTI mutations V90I, A98G, K101H, V106I, and V179D/F/I/T, and the NRTI mutations E44D, A62V, T69D, V75I, F77L, F116Y, and V118I, the prevalence of pre-existing low-frequency mutations at baseline was $15.2 \%$ overall. 
Table 1. Baseline characteristics of subjects included in deep sequencing analysis.

\begin{tabular}{|c|c|c|c|}
\hline Characteristic & $\begin{array}{c}\text { RPV/FTC/TDF RAP } \\
N=24\end{array}$ & $\begin{array}{c}\text { EFV/FTC/TDF RAP } \\
\qquad=9\end{array}$ & $\begin{array}{c}\text { Non-RAP Controls } \\
\qquad N=\mathbf{1 1 8}\end{array}$ \\
\hline Median age, years (IQR) & $37(31,45)$ & $28(22,33)$ & $40(31,47)$ \\
\hline Male, \% & $96 \%$ & $89 \%$ & $91 \%$ \\
\hline White race, $\%$ & $50 \%$ & $44 \%$ & $62 \%$ \\
\hline $\begin{array}{l}\text { Mean baseline CD4 cell } \\
\text { count, cells } / \mathrm{mm}^{3}(\mathrm{SD})\end{array}$ & 253 (199) & $370(175)$ & $329(206)$ \\
\hline $\begin{array}{l}\text { Mean baseline HIV-1 RNA, } \\
\log _{10} \text { copies } / \mathrm{mL} \text { (SD) }\end{array}$ & $5.9(6.2)$ & $5.4(5.7)$ & $5.7(6.1)$ \\
\hline$\leqslant 100,000$ copies $/ \mathrm{mL}, \mathrm{n}(\%)$ & $11(46 \%)$ & $5(56 \%)$ & $52(44 \%)$ \\
\hline$>100,000$ copies $/ m L, n(\%)$ & $13(54 \%)$ & $4(44 \%)$ & $66(56 \%)$ \\
\hline \multicolumn{4}{|l|}{ HIV-1 Subtype, n (\%) } \\
\hline A & 0 & 0 & $1(0.85 \%)$ \\
\hline $\mathrm{AE}$ & 0 & 0 & $3(2.54 \%)$ \\
\hline AG & 0 & 0 & $1(0.85 \%)$ \\
\hline B & $23(95.8 \%)$ & $8(88.9 \%)$ & $102(86.4 \%)$ \\
\hline $\mathrm{C}$ & 0 & 0 & $3(2.54 \%)$ \\
\hline $\mathrm{D}$ & 0 & 0 & $1(0.85 \%)$ \\
\hline $\mathrm{G}$ & 0 & 0 & $2(1.70 \%)$ \\
\hline Complex & $1(4.2 \%)$ & $1(11.1 \%)$ & $5(2.54 \%)$ \\
\hline
\end{tabular}

Table 2. Number of subjects with primary NRTI and NNRTI drug resistance mutations detected at low frequency ( $\geqslant 2 \%$ to $20 \%$ ) in baseline samples by deep sequencing.

\begin{tabular}{ccccc}
\hline & $\begin{array}{c}\text { RPV/FTC/TDF } \\
\text { RAP N = 24 }\end{array}$ & $\begin{array}{c}\text { EFV/FTC/TDF } \\
\text { RAP N =9 }\end{array}$ & $\begin{array}{c}\text { Non-RAP Controls } \\
\mathbf{N}=\mathbf{1 1 8}\end{array}$ & Total N = 151 \\
\hline No mutations, n (\%) & $24(100 \%)$ & $9(100 \%)$ & $108(91.5 \%)$ & $141(93.4 \%)$ \\
Any NRTI-R a or & 0 & 0 & $10(8.5 \%)$ & $10(6.6 \%)$ \\
NNRTI-R b n (\%) & 0 & 0 & $3(2.5 \%)$ & $3(2.0 \%)$ \\
Any NRTI-R, n (\%) & 0 & 0 & $7(5.9 \%)$ & $7(4.6 \%)$ \\
\hline Any NNRTI-R, n (\%) & 0 & 0 & 0
\end{tabular}

a Primary nucleoside/nucleotide reverse transcriptase inhibitor resistance (NRTI-R) mutations are M41L, K65N/R, D67N, T69 insertion/deletion, K70E/R, L74V/I, Y115F, Q151M, M184I/V, L210W, T215Y/F, and $\mathrm{K} 219 \mathrm{E} / \mathrm{Q} / \mathrm{N} / \mathrm{R}$ in RT; ${ }^{\mathrm{b}}$ Primary non-nucleoside reverse transcriptase inhibitor resistance (NNRTI-R) mutations are L100I, K101E/P, K103N/S, V106A/M, V108I, E138A/G/K/Q/R, V179L, Y181C/I/V, Y188C/H/L, G190A/E/Q/S, H221Y, P225H, F227C, and M230I/L in RT.

Most non-RAP control subjects (eight out of 10) with primary NRTI and NNRTI drug resistance mutations present at low frequency at baseline were virologic successes (HIV-1 RNA $<50$ copies $/ \mathrm{mL}$ ) by the FDA snapshot algorithm at Week 96 (Table 3). The two subjects without virologic success in the EFV/FTC/TDF arm with low-frequency NRTI resistance mutations were lost to follow up after an initial virologic response.

In addition to minority species, primary NRTI or NNRTI drug resistance mutations present at levels $>20 \%$ by deep sequencing were detected in baseline samples from four non-RAP control subjects and one RAP subject. None of the mutations detected were study protocol exclusion mutations. In the non-RAP control group, one subject in the EFV / FTC/TDF arm had D67N and K219Q detected at $>99 \%$ frequency by deep sequencing. These same mutations were detected by population sequencing at screening and the subject was a virologic success at Week 96 . Three additional control subjects had V108I detected at baseline. Two of these subjects (V108I at 30.4\% and $98.8 \%$ by deep sequencing) also had V108I detected by population sequencing at screening and were virologic successes at Week 96 (one in each treatment arm). The third subject had V108I at 79.6\% by deep sequencing at baseline that was not detected by population sequencing at screening. This subject was in the EFV/FTC/TDF arm and was dosed, but withdrew consent after a single day on study and therefore had no data in the Week 96 visit window. One RPV/FTC/TDF RAP subject had K219R at 99\% by deep sequencing at 
baseline; this subject also had K219R present by population sequencing at screening, baseline, and virologic failure.

Table 3. Primary NRTI and NNRTI drug resistance mutations detected at low frequency ( $\geqslant 2 \%$ to $20 \%$ ) in baseline samples from control subjects by deep sequencing and Week 96 snapshot outcome.

\begin{tabular}{|c|c|c|c|c|c|}
\hline $\begin{array}{c}\text { Subject } \\
\text { Number }\end{array}$ & $\begin{array}{c}\text { Baseline HIV-1 } \\
\text { RNA (Copies/mL) }\end{array}$ & $\begin{array}{c}\text { NRTI-R }{ }^{b} \\
\text { (\% Frequency) }\end{array}$ & $\begin{array}{c}\text { NNRTI-R }{ }^{\mathrm{c}} \\
\text { (\% Frequency) }\end{array}$ & $\begin{array}{l}\text { Mutant Copy } \\
\text { Number }\end{array}$ & $\begin{array}{l}\text { Week } 96 \text { Snapshot } \\
\text { Outcome }\end{array}$ \\
\hline \multicolumn{6}{|c|}{ RPV/FTC/TDF } \\
\hline 1 & $3,620,000$ & K65R (2.00) & - & 72,400 & Success \\
\hline 2 & 562,000 & - & V108I (2.23) & 12,533 & Success \\
\hline 3 & 535,000 & - & E138K (2.11) & 11,289 & Success \\
\hline 4 & $1,240,000$ & - & Y181C (2.87) & 35,588 & Success \\
\hline 5 & 92,200 & - & G190E (3.73) & 3,439 & Success \\
\hline 6 & 527,000 & - & M230I (5.31) & 27,984 & Success \\
\hline \multicolumn{6}{|c|}{ EFV/FTC/TDF } \\
\hline 7 & 61,400 & M41L (12.3) & - & 7,552 & $\underset{\mathrm{d}}{\text { No data in window }}$ \\
\hline 8 & $2,500,000$ & K219Q (2.36) & - & 59,000 & Failure e \\
\hline 9 & 164,000 & - & L100I (14.6) & 23,944 & Success \\
\hline 10 & 93,000 & - & G190E (2.33) & 2,167 & Success \\
\hline
\end{tabular}

-, no mutations. ${ }^{a}$ All subjects had HIV-1 subtype $\mathrm{B} ;{ }^{\mathrm{b}}$ Primary nucleoside/nucleotide reverse transcriptase inhibitor resistance (NRTI-R) mutations are M41L, K65N/R, D67N, T69 insertion/deletion, K70E/R, L74V/I, Y115F, Q151M, M184I/V, L210W, T215Y/F, and K219E/Q/N/R in RT; ${ }^{c}$ Primary non-nucleoside reverse transcriptase inhibitor resistance (NNRTI-R) mutations are L100I, K101E/P, K103N/S, V106A/M, V108I, E138A/G/K/Q/R, V179L, Y181C/I/V, Y188C/H/L, G190A/E/Q/S, H221Y, P225H, F227C, and M230I/L in $\mathrm{RT}^{\mathrm{d}}{ }^{\mathrm{S}}$ Subject was lost to follow-up. HIV-1 RNA at last visit on study (Week 84 ) was $<50$ copies $/ \mathrm{mL}$; ${ }^{\mathrm{e}}$ Subject was lost to follow-up. HIV-1 RNA at last visit on study (Week 16) was 207 copies/mL.

Deep sequencing of samples from the failure time point in RAP subjects was generally consistent with the population sequencing results from the same time point (Table 4). Deep sequencing detected additional primary NRTI and NNRTI drug resistance mutations that were not detected by population sequencing in five RPV/FTC/TDF RAP subjects and two EFV/FTC/TDF RAP subjects. Most of these mutations were present at low frequency ( $\geqslant 2 \%$ to $20 \%$ ) and also contained mutations of the same drug class present at levels $>20 \%$ that were detected by population sequencing.

Table 4. Primary NRTI and NNRTI drug resistance mutations detected in virologic failure samples from RAP subjects by deep sequencing compared with population sequencing.

\begin{tabular}{|c|c|c|c|c|c|c|}
\hline \multirow[b]{2}{*}{$\begin{array}{l}\text { Subject } \\
\text { Number }\end{array}$} & \multirow{2}{*}{$\begin{array}{c}\text { Baseline } \\
\text { HIV-1 RNA } \\
\text { (copies/mL) }\end{array}$} & \multirow{2}{*}{$\begin{array}{c}\text { HIV-1 RNA } \\
\text { at VF } \\
\text { (copies/mL) }\end{array}$} & \multicolumn{2}{|c|}{ Deep Sequencing } & \multicolumn{2}{|c|}{ Population Sequencing } \\
\hline & & & $\begin{array}{c}\text { NRTI-R }{ }^{\mathrm{a}} \\
\text { (\% Frequency) }\end{array}$ & $\begin{array}{c}\text { NNRTI-R }^{b} \\
\text { (\% Frequency) }\end{array}$ & NRTI-R ${ }^{a}$ & NNRTI-R ${ }^{b}$ \\
\hline \multicolumn{7}{|c|}{ RPV/FTC/TDF } \\
\hline 1 & $2,330,000$ & 586,000 & M184V (99) & $\begin{array}{l}\text { K101E (64) } \\
\text { E138K (11) } \\
\text { Y181I (85) }\end{array}$ & M184V & $\begin{array}{l}\text { K101K/E } \\
\text { Y181I }\end{array}$ \\
\hline 2 & $2,140,000$ & 824,000 & $\frac{\text { D67N (2.4) }}{\text { M184I (>99) }}$ & $\begin{array}{l}\text { E138K (96) } \\
\text { E138Q (4) } \\
\text { H221Y (49) }\end{array}$ & M184I & $\begin{array}{c}\text { E138K } \\
\mathrm{H} 221 \mathrm{H} / \mathrm{Y}\end{array}$ \\
\hline 3 & 98,900 & 9,370 & $\frac{\text { D67N (62) }}{\text { M184V (35) }}$ & - & $\frac{K 65 K / R}{\mathrm{M} 184 \mathrm{M} / \mathrm{I} / \mathrm{V}}$ & - \\
\hline 4 & 63,000 & 343,000 & $\begin{array}{l}\text { M184V (>99) } \\
\text { K219E (5.7) } \\
\text { K219N (5.6) } \\
\end{array}$ & Y181C (>99) & M184V & Y181C \\
\hline 5 & 12,400 & 70,600 & $\begin{array}{l}\text { M184I (>99) } \\
\text { K219R (>99) }\end{array}$ & $\begin{array}{l}\text { E138K (34) } \\
\text { E138Q (66) } \\
\text { H221Y (34) }\end{array}$ & $\begin{array}{c}\text { M184I } \\
\text { K219R d }\end{array}$ & E138K/Q \\
\hline
\end{tabular}


Table 4. Cont.

\begin{tabular}{|c|c|c|c|c|c|c|}
\hline \multirow[b]{2}{*}{$\begin{array}{l}\text { Subject } \\
\text { Number }\end{array}$} & \multirow{2}{*}{$\begin{array}{c}\text { Baseline } \\
\text { HIV-1 RNA } \\
\text { (copies/mL) }\end{array}$} & \multirow{2}{*}{$\begin{array}{c}\text { HIV-1 RNA } \\
\text { at VF } \\
\text { (copies/mL) }\end{array}$} & \multicolumn{2}{|c|}{ Deep Sequencing } & \multicolumn{2}{|c|}{ Population Sequencing } \\
\hline & & & $\begin{array}{c}\text { NRTI-R }{ }^{\mathrm{a}} \\
\text { (\% Frequency) }\end{array}$ & $\begin{array}{l}\text { NNRTI-R } \\
\text { (\% Frequency) }\end{array}$ & NRTI-R ${ }^{a}$ & NNRTI-R ${ }^{b}$ \\
\hline 6 & $6,410,000$ & $1,530,000$ & M184I (>99) & $\begin{array}{l}\text { K101E (58) } \\
\text { E138K (45) } \\
\text { Y181C (44) } \\
\text { H221Y (42) }\end{array}$ & M184I & $\begin{array}{l}\mathrm{K} 101 \mathrm{~K} / \mathrm{E} \\
\mathrm{E} 138 \mathrm{E} / \mathrm{K} \\
\mathrm{Y} 181 \mathrm{Y} / \mathrm{C} \\
\mathrm{H} 221 \mathrm{H} / \mathrm{Y}\end{array}$ \\
\hline 7 & $2,510,000$ & 20,500 & $\begin{array}{c}\text { K65R (>99) } \\
\text { T69del (24) } \\
\text { K219E (>99) }\end{array}$ & $\begin{array}{l}\text { Y181C (99) } \\
\text { Y188H (99) }\end{array}$ & $\begin{array}{c}\text { K65R } \\
\text { T69T/del } \\
\text { K219K/E }\end{array}$ & $\begin{array}{l}\text { Y181C } \\
\text { Y188H }\end{array}$ \\
\hline 8 & 891,000 & 46,600 & M184V (>99) & $\begin{array}{l}\text { K101E (18) } \\
\text { Y181I (>99) }\end{array}$ & M184V & $\begin{array}{c}\text { K101K/E } \\
\text { Y181I }\end{array}$ \\
\hline 9 & 621,000 & 409,000 & M184V (>99) & $\begin{array}{l}\text { E138K (>99) } \\
\text { Y181I (>99) }\end{array}$ & M184V & E138K Y181I \\
\hline 10 & 564,000 & 24,000 & M184I (>99) & E138Q (>99) & M184I & E138K/Q \\
\hline 11 & 288,000 & 36,500 & $\begin{array}{l}\text { Y115F (>99) } \\
\text { M184I (99) } \\
\text { K219E (69) }\end{array}$ & K101E (99) & $\begin{array}{c}\text { Y115Y/F } \\
\text { M184I } \\
\text { K219K/E }\end{array}$ & $\begin{array}{c}\text { K101E } \\
Y 181 Y / C \\
\end{array}$ \\
\hline 12 & 215,000 & 24,100 & M184I (>99) & $\begin{array}{l}\text { E138K (>99) } \\
\text { H221Y (6.1) }\end{array}$ & M184I & $\begin{array}{c}\text { E138K } \\
\mathrm{H} 221 \mathrm{H} / \mathrm{Y}\end{array}$ \\
\hline 13 & 127,000 & 1,120 & $\begin{array}{c}\text { K70E (>99) } \\
\text { M184I (>99) }\end{array}$ & E138K (>99) & $\begin{array}{c}\text { K70K/E } \\
\text { M184I }\end{array}$ & E138K \\
\hline 14 & 65,100 & 489 & M184V (64) & $\begin{array}{l}\text { V108I (61) } \\
\text { F227C (62) }\end{array}$ & $\frac{K 65 K / N}{\mathrm{M} 184 \mathrm{M} / \mathrm{I} / \mathrm{V}}$ & $\begin{array}{l}\mathrm{V} 108 \mathrm{~V} / \mathrm{I} \\
\mathrm{F} 227 \mathrm{~F} / \mathrm{C} \\
\mathrm{M} 230 \mathrm{M} / \mathrm{I} \\
\end{array}$ \\
\hline 15 & 314,000 & 998 & M184I (>99) & - & M184I & 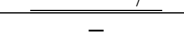 \\
\hline 16 & 59,100 & 2,750 & M184I (>99) & E138K (>99) & M184I & E138K \\
\hline 17 & 50,700 & 798 & M184I (>99) & $\begin{array}{l}\text { E138K (>99) } \\
\text { H221Y (40) }\end{array}$ & M184I & $\begin{array}{c}\text { E138K } \\
\mathrm{H} 221 \mathrm{H} / \mathrm{Y}\end{array}$ \\
\hline 18 & 50,600 & 2,570 & $\begin{array}{c}\text { L74V (>99) } \\
\text { M184V (>99) }\end{array}$ & $\begin{array}{l}\text { L100I (>99) } \\
\text { K103N (99) } \\
\text { P225H (99) }\end{array}$ & L74V M184V & $\begin{array}{c}\text { L100I K103N } \\
\text { P225H }\end{array}$ \\
\hline 19 & 44,400 & 7,590 & $\begin{array}{l}\text { M184I (>99) } \\
\text { K219E (>99) }\end{array}$ & $\begin{array}{c}\text { K101E (43) } \\
\text { Y181C (>99) } \\
\text { M230L (>99) }\end{array}$ & $\begin{array}{l}\text { M184I } \\
\text { K219E }\end{array}$ & $\begin{array}{l}\text { K101E } \\
\text { Y181C } \\
\text { M230L } \\
\end{array}$ \\
\hline 20 & 10,500 & 1,980 & M184I (>99) & E138K (>99) & M184I & E138K \\
\hline 21 & 522,000 & 527 & $\mathrm{AF}$ & $\mathrm{AF}$ & - & - \\
\hline 22 & 60,600 & 518 & $\mathrm{AF}$ & $\mathrm{AF}$ & - & - \\
\hline 23 & 154,000 & 659 & - & - & - & - \\
\hline 24 & 20,600 & 24,700 & - & - & - & - \\
\hline & & & EFV/FTC/T & & & \\
\hline 1 & $1,440,000$ & 192,000 & $\begin{array}{c}\text { K65R (7.8) } \\
\text { D67N (99) } \\
\text { M184I (>99) } \\
\text { K219E (50) } \\
\text { K219N (2.4) }\end{array}$ & $\begin{array}{l}\text { G190E (55) } \\
\text { G190Q (44) } \\
\text { H221Y (8.7) } \\
\end{array}$ & $\begin{array}{c}\text { D67N M184I } \\
\text { K219K/E }\end{array}$ & G190E/Q \\
\hline 2 & 150,000 & 485 & - & M230L (>99) & - & - \\
\hline 3 & 97,700 & 648 & M184I (>99) & M230L (>99) & M184M/I & M230M/L \\
\hline 4 & 32,400 & 24,700 & - & K103N (99) & - & K103N \\
\hline 5 & 11,200 & 3,020 & - & Y188L (>99) & - & Y188L \\
\hline 6 & 452,000 & 46,600 & - & - & - & - \\
\hline 7 & 161,000 & 205,000 & - & - & - & - \\
\hline 8 & 22,500 & 20,100 & - & - & - & - \\
\hline 9 & 22,100 & 2,020 & - & - & - & - \\
\hline
\end{tabular}

- , no mutations. AF, assay failure; VF, virologic failure. Mutations detected by deep sequencing but absent by population sequencing are underlined and bolded. Mutations detected by population sequencing but absent by deep sequencing are underlined and italicized. ${ }^{a}$ Primary nucleoside/nucleotide reverse transcriptase inhibitor resistance (NRTI-R) mutations are M41L, K65N/R, D67N, T69 insertion/deletion, K70E/R, L74V/I, Y115F, Q151M, M184I/V, L210W, T215Y/F, and K219E/Q/N/R in RT; ${ }^{b}$ Primary non-nucleoside reverse transcriptase inhibitor resistance (NNRTI-R) mutations are L100I, K101E/P, K103N/S, V106A/M, V108I, E138A/G/K/Q/R, V179L, Y181C/I/V, Y188C/H/L, G190A/E/Q/S, H221Y, P225H, F227C, and M230I/L in RT; ' Subject had a complex mixture of HIV-1 subtypes. All other subjects had HIV-1 subtype B; ${ }^{\mathrm{d}}$ Subject also had K219R present by population sequencing at the screening and baseline visits. 
Three samples with mutant frequencies of $>20 \%$ had discrepant results for deep sequencing and population sequencing at the virologic failure time point. In two cases, the discrepancies occurred in subjects with HIV- 1 RNA $<500$ copies/mL (less than the validated limit of detection for both assays) in the virologic failure sample. EFV/FTC/TDF RAP subject \#2 had HIV-1 RNA 485 copies/mL with M230L detected by deep sequencing at $>99 \%$ frequency, but had no mutations detected by population sequencing. RPV/FTC/TDF RAP subject \#14 had HIV-1 RNA 489 copies/mL with M184V (63\%) and F227C (62\%) detected by deep sequencing and K65K/N, M184M/I/V, V108V/I, F227F/C, and M230M/I present by population sequencing. RPV/FTC/TDF RAP subject \#3 had HIV-1 RNA 9370 copies / $\mathrm{mL}$ with D67N (62\%) and M184V (35\%) detected by deep sequencing and K65K/R and $\mathrm{M} 184 \mathrm{M} / \mathrm{I} / \mathrm{V}$ present by population sequencing.

\section{Discussion}

Baseline HIV-1 primary NNRTI- and NRTI-resistance associated mutations were detected as minority populations (frequencies $>2 \%$ and $\leqslant 20 \%$ ) in $6.6 \%$ of subject isolates analyzed by deep sequencing in the STaR study. The prevalence of low-frequency drug resistance species in the current study is slightly lower than that observed in other studies of treatment-naïve patients $[3,4,10,23]$. This difference may be due in part to the use of a more focused mutation list of primary NNRTI- and NRTI-resistance mutations known to have significant impact on susceptibility to the components of $\mathrm{RPV} / \mathrm{FTC} / \mathrm{TDF}$ and EFV/FTC/TDF, and did not include minor or polymorphic mutations that may have been part of mutation lists used in other studies.

No RPV /FTC/TDF or EFV/FTC/TDF-treated subjects who experienced virologic failure with resistance development had pre-existing primary NNRTI- or NRTI-resistant virus populations at levels $>2 \%$ and $\leqslant 20 \%$. While the presence of pre-existing low-frequency resistance mutations does not appear to explain the higher rate of virologic failure in high baseline viral load subjects treated with RPV/FTC/TDF observed in the STaR study, it is possible that subjects with high baseline viral load have greater viral diversity, including more virus species containing drug resistance mutations potentially present below the limit of detection of the assay used in this study, and this could impact clinical outcome. The majority of subjects (seven out of 10) who had low-frequency primary NNRTIor NRTI-resistance mutations present at baseline also had HIV-1 RNA $>100,000$ copies/mL, supporting the idea that subjects with high baseline viral load have greater variation in their virus population that pre-exists as a result of errors generated during viral replication driven by HIV-1 reverse transcriptase. However, the high virologic response rates among the few subjects with low-frequency pre-existing resistance in the STaR study suggest that other factors such as adherence, CD4 cell count, or drug exposure are playing a more significant role in determining virologic outcome in high baseline viral load subjects.

All subjects with low-frequency populations of primary NNRTI- or NRTI-associated resistance mutations present at baseline in the current study had single mutations detected, which may not have been sufficient to influence virologic response. It is possible that the presence of multiple mutations and/or mutations to multiple classes of antiretroviral drugs may be needed in order for low-level mutations to increase the risk of virologic failure. However, a recent study in treatment-experienced patients found that neither single nor dual-class minority resistant viruses at baseline had a significant impact on virologic response [7]. It is also possible that particular mutations or classes of mutations may have a greater impact on clinical outcomes than others. Furthermore, our study was limited by the exclusion of subjects with NNRTI or NRTI resistance mutations present by population sequencing at screening, the potential exclusion of additional subjects with pre-existing low-frequency resistance mutations who did not meet other enrollment criteria, the limited number of subjects that developed resistance during the study, and the observation that most low-frequency mutations detected at baseline were very close to the deep sequencing assay cutoff of $2 \%$.

Previous studies have suggested a dose-dependent relationship between the absolute copy number of a particular viral mutant (or mutational load) and impact on virologic outcome [8,24]. This is thought to be particularly relevant for the NNRTI mutation K103N which has been associated 
with an increased risk of virologic failure on EFV-based regimens if present above a threshold of 2000 copies/mL [24]. Mutant copy number did not appear to play a significant role in the current study since most of the subjects with a pre-existing low-frequency mutant had HIV-1 RNA $>100,000$ copies $/ \mathrm{mL}$, resulting in a mutant copy number of greater than 2000 copies $/ \mathrm{mL}$ in all cases as well as a high virologic success rate.

Deep sequencing results were concordant with the population sequencing results in the majority of samples. Additional low-frequency primary NRTI and NNRTI drug resistance mutations were detected by deep sequencing in five virologic failure samples in which mutations of the same class had also been detected by population sequencing. In addition, there were a small number of instances (one baseline sample and three virologic failure samples) where the assays produced discrepant results for mutations with frequencies $>20 \%$. Since the deep sequencing and population sequencing assays are from different clinical laboratories and use different sets of primers for the reverse transcription and PCR steps of the assays, it is possible that each assay amplified a slightly different virus population due to differences in primer sensitivity and/or specificity. Furthermore, two of the virologic failure samples with discrepancies had HIV-1 RNA $<500$ copies $/ \mathrm{mL}$, which is below the validated limit of detection for both assays. The limited virus present in these samples may have led to increased differential amplification between the two assays and potential over- or underestimation of the variants present. All of the mutations detected by population sequencing that were not detected by deep sequencing were present as mixtures with wild-type and may have been due to the overcalling of trace signals by population sequencing.

Numerous studies have examined the effect of minority populations of drug resistance-associated virus to impact treatment outcome in treatment-naïve patients, particularly for NNRTI-based regimens. Some studies have reported an increased risk of virologic failure associated with the presence of pre-existing low-frequency drug resistant variants [2,8-10]. Others have found limited to no evidence for a role of low-frequency variants in virologic response, consistent with the results of the current study $[3,5,6,11]$. Notably, our results are in agreement with the deep sequencing analysis from subjects who failed treatment with the components of RPV/FTC/TDF in the pivotal ECHO and THRIVE studies, demonstrating that the mutations that emerged in subjects on RPV-containing regimens were not present as low-frequency variants at baseline [25]. It is possible that variants that are transmitted and present at low frequency prior to treatment initiation may have a different impact on treatment outcomes than low-frequency drug resistance virus species arising under selective drug pressure in treatment-experienced patients. The clinical significance of minority drug resistant variants requires further investigation, particularly with regard to different treatment regimens and patient populations.

Deep sequencing of HIV-1 patient isolates is currently still an experimental technique that is not routinely used in clinical practice to inform patient management as the clinical significance of low-frequency drug resistant variants remains unclear. Since deep sequencing can be cost-effective compared to population sequencing, it is possible that deep sequencing may replace population sequencing as standard of care in the future. In this case, prospective clinical data should be utilized to determine the clinical significance of variant frequencies or mutational burden by position and specific antiretroviral regimen.

Acknowledgments: This work was supported by Gilead Sciences, Inc. We would like to thank the patients, investigators, study staff, and Gilead staff who took part in the STaR study. A portion of these data was presented at the Conference for Retroviruses and Opportunistic Infections, Seattle, Washington, 2015 (abstract 605).

Author Contributions: Danielle P. Porter, Michael D. Miller, and Kirsten L. White conceived and designed the study. Martin Daeumer and Alexander Thielen performed the deep sequencing assay. Silvia Chang and Ross Martin analyzed the deep sequencing data. Cal Cohen was the principal investigator of the STaR study and provided clinical input. Danielle P. Porter analyzed the data and wrote the manuscript. All authors reviewed the manuscript.

Conflicts of Interest: Danielle P. Porter, Silvia Chang, Ross Martin, Cal Cohen, Michael D. Miller, and Kirsten L. White are employees and shareholders of Gilead Sciences, Inc. Martin Daeumer and Alexander Thielen have no conflicts of interest. 


\section{References}

1. Department for Health and Human Services (DHHS). Guidelines for the Use of Antiretroviral Agents in HIV-1-Infected Adults and Adolescents. Developed by the Dhhs Panel on Antiretroviral Guidelines for Adults and Adolescents-A Working Group of the Office of Aids Research Advisory Council (OARAC). Available online: http:/ / aidsinfo.Nih.Gov/guidelines (accessed on 5 November 2015).

2. Li, J.Z.; Paredes, R.; Ribaudo, H.J.; Kozal, M.J.; Svarovskaia, E.S.; Johnson, J.A.; Geretti, A.M.; Metzner, K.J.; Jakobsen, M.R.; Hullsiek, K.H.; et al. Impact of minority nonnucleoside reverse transcriptase inhibitor resistance mutations on resistance genotype after virologic failure. J. Infect. Dis. 2013, 207, 893-897. [CrossRef] [PubMed]

3. Nicot, F.; Saune, K.; Raymond, S.; Jeanne, N.; Carcenac, R.; Lefebvre, C.; Cuzin, L.; Marchou, B.; Delobel, P.; Izopet, J. Minority resistant HIV-1 variants and the response to first-line NNRTI therapy. J. Clin. Virol. 2015, 62, 20-24. [CrossRef] [PubMed]

4. Lataillade, M.; Chiarella, J.; Yang, R.; Schnittman, S.; Wirtz, V.; Uy, J.; Seekins, D.; Krystal, M.; Mancini, M.; McGrath, D.; et al. Prevalence and clinical significance of HIV drug resistance mutations by ultra-deep sequencing in antiretroviral-naive subjects in the CASTLE study. PLoS ONE 2010, 5, e10952. [CrossRef] [PubMed]

5. Messiaen, P.; Verhofstede, C.; Vandenbroucke, I.; Dinakis, S.; van Eygen, V.; Thys, K.; Winters, B.; Aerssens, J.; Vogelaers, D.; Stuyver, L.J.; et al. Ultra-deep sequencing of HIV-1 reverse transcriptase before start of an NNRTI-based regimen in treatment-naive patients. Virology 2012, 426, 7-11. [CrossRef] [PubMed]

6. Metzner, K.J.; Scherrer, A.U.; von Wyl, V.; Boni, J.; Yerly, S.; Klimkait, T.; Aubert, V.; Furrer, H.; Hirsch, H.H.; Vernazza, P.L.; et al. Limited clinical benefit of minority K103N and Y181C-variant detection in addition to routine genotypic resistance testing in antiretroviral therapy-naive patients. AIDS 2014, 28, 2231-2239. [CrossRef] [PubMed]

7. Charpentier, C.; Lee, G.Q.; Rodriguez, C.; Visseaux, B.; Storto, A.; Fagard, C.; Molina, J.M.; Katlama, C.; Yazdanpanah, Y.; Harrigan, P.R.; et al. Highly frequent HIV-1 minority resistant variants at baseline of the ANRS 139 TRIO trial had a limited impact on virological response. J. Antimicrob. Chemother. 2015, 70, 2090-2096. [CrossRef] [PubMed]

8. Li, J.Z.; Paredes, R.; Ribaudo, H.J.; Svarovskaia, E.S.; Metzner, K.J.; Kozal, M.J.; Hullsiek, K.H.; Balduin, M.; Jakobsen, M.R.; Geretti, A.M.; et al. Low-frequency HIV-1 drug resistance mutations and risk of NNRTI-based antiretroviral treatment failure: A systematic review and pooled analysis. JAMA 2011, 305, 1327-1335. [CrossRef] [PubMed]

9. Cozzi-Lepri, A.; Noguera-Julian, M.; di Giallonardo, F.; Schuurman, R.; Daumer, M.; Aitken, S.; Ceccherini-Silberstein, F.; D’Arminio Monforte, A.; Geretti, A.M.; Booth, C.L.; et al. Low-frequency drug-resistant HIV-1 and risk of virological failure to first-line NNRTI-based art: A multicohort European case-control study using centralized ultrasensitive 454 pyrosequencing. J. Antimicrob. Chemother. 2015, 70, 930-940. [CrossRef] [PubMed]

10. Simen, B.B.; Simons, J.F.; Hullsiek, K.H.; Novak, R.M.; Macarthur, R.D.; Baxter, J.D.; Huang, C.; Lubeski, C.; Turenchalk, G.S.; Braverman, M.S.; et al. Low-abundance drug-resistant viral variants in chronically HIV-infected, antiretroviral treatment-naive patients significantly impact treatment outcomes. J. Infect. Dis. 2009, 199, 693-701. [CrossRef] [PubMed]

11. Gianella, S.; Delport, W.; Pacold, M.E.; Young, J.A.; Choi, J.Y.; Little, S.J.; Richman, D.D.; Kosakovsky Pond, S.L.; Smith, D.M. Detection of minority resistance during early HIV-1 infection: Natural variation and spurious detection rather than transmission and evolution of multiple viral variants. J. Virol. 2011, 85, 8359-8367. [CrossRef] [PubMed]

12. Cohen, C.; Wohl, D.; Arribas, J.R.; Henry, K.; van Lunzen, J.; Bloch, M.; Towner, W.; Wilkins, E.; Ebrahimi, R.; Porter, D.; et al. Week 48 results from a randomized clinical trial of rilpivirine/emtricitabine/tenofovir disoproxil fumarate vs. Efavirenz/emtricitabine/tenofovir disoproxil fumarate in treatment-naive HIV-1-infected adults. AIDS 2014, 28, 989-997. [CrossRef] [PubMed]

13. Cohen, C.; Wohl, D.; Arribas, J.; Henry, K.; van Lunzen, J.; Bloch, M.; Towner, W.; Wilkins, E.; Ebrahimi, R.; Porter, D.; et al. Star study: Single-tablet regimen rilpivirine/emtricitabine/tenofovir DF maintains non-inferiority to efavirenz/emtricitabine/tenofovir DF in art-naïve adultsweek 96 results. In Proceedings of the 14th European AIDS Conference, Brussels, Belgium, 16-19 October 2013. 
14. Porter, D.P.; Kulkarni, R.; Fralich, T.; Miller, M.D.; White, K.L. 96-Week resistance analyses of the star study: Rilpivirine/emtricitabine/tenofovir df vs. efavirenz/emtricitabine/tenofovir DF in antiretroviral-naive, HIV-1-infected subjects. HIV Clin. Trials 2015, 16, 30-38. [CrossRef] [PubMed]

15. Gilead Sciences Inc. Complera ${ }^{\circledR}$ (emtricitabine, rilpivirine, tenofovir disoproxil fumarate) tablets. Us prescribing information. Foster city, ca. Revised May 2015.

16. Gilead Sciences International Limited. Eviplera ${ }^{\circledR}$ (emtricitabine, rilpivirine, tenofovir disoproxil fumarate) $200 \mathrm{mg} / 25 \mathrm{mg} / 245 \mathrm{mg}$ film-coated tablets. Summary of product characteristics. Cambridge, UK, May 2015.

17. U.S. Department of Health and Human Services; Food and Drug Administration (FDA); Center for Drug Evaluation and Research (CDER). Human Immunodeficiency Virus-1 Infection: Developing Antiretroviral Drugs for Treatment. Guidance for Industry. Revision 1. Available online: http://www.fda.gov/ downloads/Drugs/GuidanceComplianceRegulatoryInformation/Guidances/UCM355128.pdf (accessed on 1 December 2015).

18. Porter, D.P.; Kulkarni, R.; Fralich, T.; Miller, M.D.; White, K.L. Characterization of HIV-1 drug resistance development through week 48 in antiretroviral naive subjects on rilpivirine/emtricitabine/tenofovir DF or efavirenz/emtricitabine/tenofovir DF in the STaR study (GS-US-264-0110). J. Acquir. Immune Defic. Syndr. 2014, 65, 318-326. [CrossRef] [PubMed]

19. Thielen, A.; Martini, N.; Thiele, B.; Daumer, M. Validation of HIV-1 drug resistance testing by deep sequencing: Insights from comparative Sanger sequencing. In Proceedings of the International Workshop on Antiviral Drug Resistance (IDRW), Berlin, Germany, 3-7 June 2014.

20. Yang, X.; Charlebois, P.; Gnerre, S.; Coole, M.G.; Lennon, N.J.; Levin, J.Z.; Qu, J.; Ryan, E.M.; Zody, M.C.; Henn, M.R. De novo assembly of highly diverse viral populations. BMC Genom. 2012, 13. [CrossRef] [PubMed]

21. Lee, W.P.; Stromberg, M.P.; Ward, A.; Stewart, C.; Garrison, E.P.; Marth, G.T. Mosaik: A hash-based algorithm for accurate next-generation sequencing short-read mapping. PLoS ONE 2014, 9, e90581. [CrossRef] [PubMed]

22. Petropoulos, C.J.; Parkin, N.T.; Limoli, K.L.; Lie, Y.S.; Wrin, T.; Huang, W.; Tian, H.; Smith, D.; Winslow, G.A.; Capon, D.J.; et al. A novel phenotypic drug susceptibility assay for human immunodeficiency virus type 1. Antimicrob. Agents Chemother. 2000, 44, 920-928. [CrossRef] [PubMed]

23. Nicot, F.; Saliou, A.; Raymond, S.; Saune, K.; Dubois, M.; Massip, P.; Marchou, B.; Delobel, P.; Izopet, J. Minority variants associated with resistance to HIV-1 nonnucleoside reverse transcriptase inhibitors during primary infection. J. Clin. Virol. 2012, 55, 107-113. [CrossRef] [PubMed]

24. Goodman, D.D.; Zhou, Y.; Margot, N.A.; McColl, D.J.; Zhong, L.; Borroto-Esoda, K.; Miller, M.D.; Svarovskaia, E.S. Low level of the K103N HIV-1 above a threshold is associated with virological failure in treatment-naive individuals undergoing efavirenz-containing therapy. AIDS 2011, 25, 325-333. [CrossRef] [PubMed]

25. Van Eygen, V.; Thys, K.; Vingerhoets, J.; van Hove, C.; Rimsky, L.T.; Aerssens, J.; Picchio, G.R. Deep sequencing analysis of rilpivirine virologic failures in the phase III studies echo and thrive shows no association with the presence of minority resistance-associated variants at baseline. In Proceedings of the International Workshop on HIV \& Hepatitis Virus Drug Resistance and Curative Strategies, Melia Sitges, Spain, 5-9 June 2012.

(C) 2015 by the authors; licensee MDPI, Basel, Switzerland. This article is an open access article distributed under the terms and conditions of the Creative Commons by Attribution (CC-BY) license (http://creativecommons.org/licenses/by/4.0/). 